\title{
FtsW exhibits distinct processive motions driven by either septal cell wall synthesis or FtsZ treadmilling in E. coli
}

\author{
Xinxing Yang ${ }^{1, \bowtie}$, Ryan McQuillen ${ }^{1}$, Zhixin Lyu ${ }^{1}$, Polly Phillips-Mason ${ }^{2}$, Ana De La Cruz ${ }^{1}$, Joshua W. McCausland ${ }^{1}$, Hai \\ Liang $^{3,5}$, Kristem E. DeMeester ${ }^{3}$, Cintia C. Santiago ${ }^{3}$, Catherine L. Grimes ${ }^{3,4}$, Piet de Boer ${ }^{2, \bigotimes}$, and Jie Xiao ${ }^{1, \square}$ \\ ${ }^{1}$ Department of Biophysics and Biophysical Chemistry, Johns Hopkins School of Medicine, Baltimore, Maryland, 21205, USA. \\ ${ }^{2}$ Department of Molecular Biology and Microbiology, School of Medicine, Case Western Reserve University, Cleveland, Ohio 44106-4960, USA. \\ ${ }^{3}$ Department of Chemistry and Biochemistry, University of Delaware, 134 Brown Lab, Newark, Delaware 19716, USA. \\ ${ }^{4}$ Department of Biological Sciences, University of Delaware, Newark, Delaware 19716, USA. \\ ${ }^{5}$ Current address: Dermatology Branch, National Institute of Arthritis and Musculoskeletal and Skin Diseases, NIH, Bethesda, MD 20892, USA
}

\begin{abstract}
During bacterial cell division, synthesis of new septal peptidoglycan (sPG) is crucial for successful cytokinesis and cell pole morphogenesis. FtsW, a SEDS (Shape, Elongation, Division and Sporulation) family protein and an indispensable component of the cell division machinery in all walled bacterial species, was recently identified in vitro as a new monofunctional peptidoglycan glycosyltransferase (PGTase). FtsW and its cognate monofunctional transpeptidase (TPase) class $B$ penicillin binding protein (PBP3 or FtsI in E. coli) may constitute the essential, bifunctional sPG synthase specific for new SPG synthesis. Despite its importance, the septal PGTase activity of FtsW has not been documented in vivo. How its activity is spatiotemporally regulated in vivo has also remained unknown. Here we investigated the septal PGTase activity and dynamics of FtsW in $E$. coli cells using a combination of single-molecule imaging and genetic manipulations. We show that FtsW exhibits robust activity to incorporate an $\mathrm{N}$-acetylmuramic acid analog at septa in the absence of other known PGTases, confirming FtsW as the essential septum-specific PGTase in vivo. Notably, we identified two populations of processive moving FtsW molecules at septa. A fast-moving population is driven by the treadmilling dynamics of FtsZ and independent of SPG synthesis. A slow-moving population is driven by active SPG synthesis and independent of FtsZ's treadmilling dynamics. We further identified that FtsN, a potential sPG synthesis activator, plays an important role in promoting the slow-moving, sPG synthesis-dependent population. Our results support a two-track model, in which inactive sPG synthase molecules follow the fast treadmilling "Z-track" to be distributed along the septum; FtsN promotes their release from the "Z-track" to become active in SPG synthesis on the slow "sPG-track". This model explains how the spatial information is integrated into the regulation of SPG synthesis activity and suggests a new mechanistic framework for the spatiotemporal coordination of bacterial cell wall constriction.
\end{abstract}

FtsW | FtsZ treadmilling | sPG synthesis

Correspondence: X.Y. (xyang47@jhmi.edu), P.d.B (pad5@case.edu), J.X. (xiao@jhmi.edu)

\section{Results}

To investigate the role of FtsW in sPG synthesis in vivo, we employed a cysteine-modification inactivation assay $(1,2)$.
Based on the homology structure of $\operatorname{RodA}(3)$, we generated twelve $f t s W^{C}$ alleles that each encodes a unique cysteine residue on the periplasmic side of FtsW (Extended Data Fig. 1 and Supplementary Table 1 and 2). Amongst these, we identified $\mathrm{FtsW}^{I 302 C}$ as a promising candidate for in vivo inactivation with the cysteine-reactive reagent MTSES (2-sulfonatoethyl methanethiosulfonate)(4). Cells expressing $\mathrm{FtsW}^{I 302 C}$ from the native chromosomal $\mathrm{fts} W$ locus grew with a wild-type (WT)-like doubling time and cell morphology in the absence of MTSES but grew into long chains and stopped dividing when treated with MTSES (Extended Data Fig. 2c, Supplementary Movie 1 and 2). WT parental BW25113 cells treated with MTSES did not show any appreciable cell division defect (Extended Data Fig. 2d), indicating that MTSES specifically inhibited the essential function of $\mathrm{FtsW}^{I 302 C}$. The homology structure of FtsW indicates that I302 resides in periplasmic loop 4 between transmembrane helices 7 and 8, likely near critical residues of the PGTase activity of SEDS proteins $(3,5)$.

To probe the contribution of FtsW to $\mathrm{sPG}$ synthesis in vivo, we labeled new cell wall peptidoglycan (PG) synthesis using an alkyne-modified $\mathrm{N}$-acetylmuramic acid carbohydrate derivative (alkyne-NAM). Unlike fluorescent Damino acid (FDAA) labels, which are incorporated into the peptide stem of $E$. coli $\mathrm{PG}$ through periplasmic exchange reactions(6), alkyne-NAM enters the endogenous cytoplasmic PG biosynthetic pathway and incorporates into newly linked glycan chains(7) composed of alternating units of NAM and N-acetylglucosamine (NAG). Subsequent labeling of the alkyne using a fluorophore-conjugated azide by copper catalyzed azide-alkyne cycloaddition (CuAAC) also known as "CLICK" chemistry allows for visualization and quantification of newly polymerized glycan strands(8).

In WT cells treated with or without MTSES, we observed robust alkyne-NAM labeling $(2 \mathrm{mg} / \mathrm{ml}, 30 \mathrm{~min}$, Fig. 1a, Methods) at septa. In $f t s W^{I 302 C}$ cells treated with MTSES (1mM, 30min), we observed a significant reduction in the percentage of cells showing septal labeling above the background level (from $34 \pm 4 \%$ to $22 \pm 4 \%, \mu \pm$ S.E.M., $\mathrm{N}>300$ cells, three independent repeats, Fig. 1b, orange bars, Supplementary Table 3). Furthermore, in this septal-labeled 


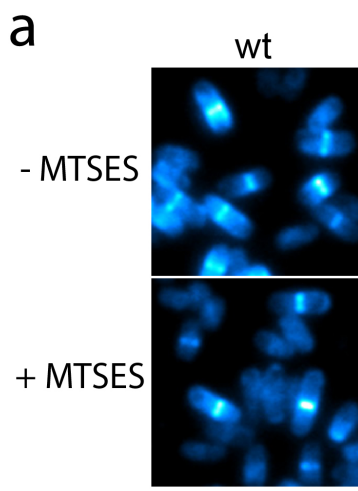

b
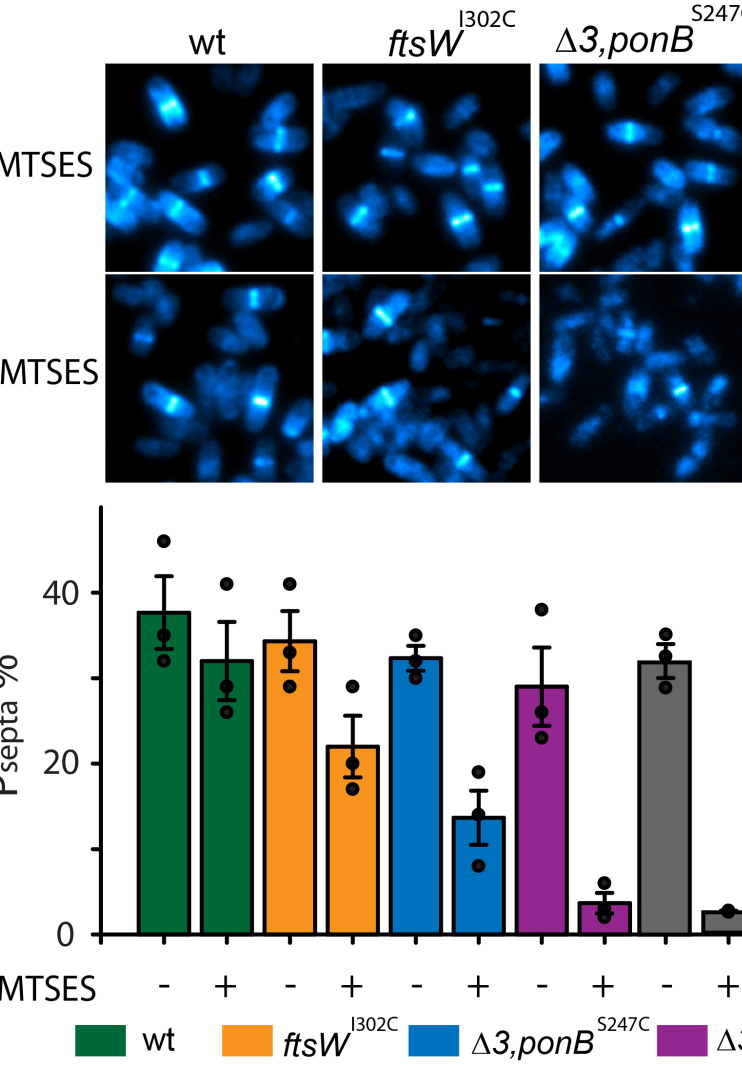

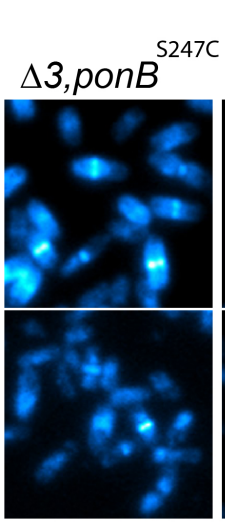

$\Delta 3, p o n B^{5247}$
$f t s W^{1302 C}$
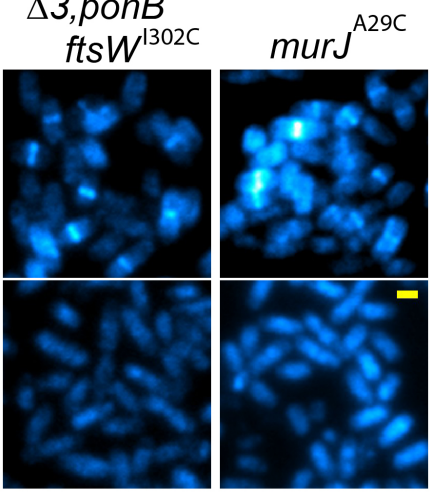

C

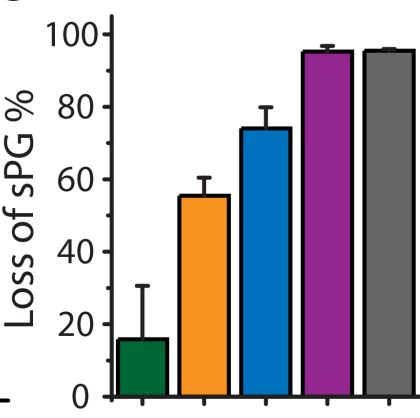

$\Delta 3, \operatorname{ponB}^{\mathrm{S} 247 \mathrm{C}} \mathrm{fts} W^{1302 C}-$ murJ $^{\mathrm{A} 29 \mathrm{C}}$

Fig. 1. FtsW is an essential septum-specific monofunctional PGTase. a Representative images of E. coli cells of different strain backgrounds labeled with AF647conjugated NAM in the absence or presence of MTSES. The contrast of each image is adjusted to allow optimized visualization of septal labeling especially for the $\Delta 3$, ponB ${ }^{S 247 C}$,ftsW ${ }^{I 302 C}$ and murJ ${ }^{A 29 C}+$ MTSES conditions. The absolute intensity is summarized in Supplementary Table 3 . Scale bar: $1 \mu$ m. b. Mean percentage of cells with septal NAM labeling above background level in the absence or presence of MTSES. c. Mean percentage of total loss in septal NAM intensity of the five strains due to MTSES. Error bars: S.E.M. of three experimental repeats (dots). Strains used were BW25113 (wt), JXY559 (ftsW ${ }^{I 302 C}$ ), HC532 ( $\Delta 3, p o n B^{S 247 C}$ ), JXY564 $\left(\Delta 3\right.$, ponB $\left.^{S 247 C}, f t s W^{I 302 C}\right)$, and JXY589 (murJ $\left.{ }^{A 29 C}\right)$, all carrying plasmid pBBR1-KU.

population of MTSES-treated $f t s W^{I 302 C}$ cells, the median fluorescence intensity dropped to $70 \pm 4 \%$ compared to that in untreated cells (Extended Data Fig. 3, Supplementary Table 3). Thus, inhibition of $\mathrm{FtsW}^{I 302 C}$ activity by MTSES caused a total reduction in septal NAM labeling of $55 \%$ (100\%-22\%/34\% $\times 70 \%$, Fig. 1c, orange bar), consistent with a major role of FtsW in septal glycan chain polymerization. The fact that significant NAM incorporation still occurred at septa of MTSES-treated $f t s W^{I 302 C}$ cells is consistent with previous reports showing that FtsW is not an essential lipid II flippase acting upstream of sPG synthesis(9) and that other PGTases contribute to septal morphogenesis as well(10). The inability of MTSES-treated $f t s W^{I 302 C}$ cells to complete cell division, even when other PGTases are still active, highlights the essential role of FtsW in successful cell wall constriction.

To further investigate the relative contributions to $\mathrm{SPG}$ polymerization by FtsW and other relevant PGTases in E. coli, we introduced the chromosomal $f t s W^{I 302 C}$ allele in a $\Delta 3$, ponB $B^{S 247 C}$ strain background1 to create a $\Delta 3$,ponB ${ }^{S 247 C}$,fts $W^{I 302 C}$ strain. The $\Delta 3$,ponB ${ }^{S 247 C}$ strain lacks the genes for PBP1A, PBP1C and MtgA, and expresses a variant (S247C) of PBP1B (PBP1B ${ }^{S 247 C}$, encoded by ponB ${ }^{S 247 C}$ ) that, like $\mathrm{FtsW}^{I 302 C}$, can be inactivated by exposure to MTSES. Untreated $\Delta 3$,ponB $B^{S 247 C}$ cells exhibited a similar percentage of labeled septa as WT cells $(32 \pm 1 \%$, $\mu \pm$ S.E.M., N $>300$ cells, three independent repeats, Fig.1b, blue bar, Supplementary Table 3). The integrated intensity at septa decreased $\sim 60 \%$ (Extended Data Fig. 3, Supplementary Table 3), however, suggesting that the PBP1B ${ }^{S 247 C}$ variant may be intrinsically less active or that PBP1A, PBP1C and/or MtgA may also contribute to sPG synthesis under our experimental condition.

When the PGTase activity of PBP1B ${ }^{S 247 C}$ in $\triangle 3$ ponB $B^{S 247 C}$ cells was inhibited by MTSES, the percentage of labeled septa dropped to $14 \pm 3 \%$ with the median intensity reduced to $65 \pm 12 \%$ of the untreated level (Fig. 1b, blue bar, Supplementary Table 3), corresponding to a total loss of septal labeling of $\sim 72 \%(100 \%-14 \% / 32 \% \times 65 \%$, Fig. $1 \mathrm{c}$, blue bar). Simultaneous inactivation of PBP1B and FtsW by MTSES in $\triangle 3$,ponB ${ }^{S 247 C}, f t s W^{I 302 C}$ cells, however, led to a background level of septal NAM labeling indistinguishable from that when the essential Lipid II flippase MurJ(9) was inhibited (Fig. 1b and c, compare purple and gray bars, Supplementary Table 3). Combined with the recent evidence that purified FtsW possesses PGTase activity(11), our results in live E.coli cells strongly support the notion that FtsW is an essential septal PGTase in vivo.

Previously, we and others showed that FtsZ's treadmilling dynamics drive the processive movement of FtsW's cognate TPase (FtsI in E. coli and PBP2B in B. sub- 
tilis) at the septum(12, 13). Such FtsZ-dependent dynamics were proposed to direct the spatial distribution of $\mathrm{sPG}$ synthesis complexes and play an important role in septum morphogenesis(12,13). Because a large body of biochemical and genetic studies indicates that FtsW associates with FtsI to form a bifunctional sPG synthase complex(11,14-16), we investigated whether FtsW exhibited similar processive movement at septa using single molecule tracking (SMT).

We constructed a C-terminal fusion of FtsW with the red fluorescent protein TagRFP-T (Supplementary Table 1) and refer to the fusion protein as FtsW-RFP for simplicity. We verified that upon replacement of chromosomal $f t s W$ with the $f t s W$-rfp allele (strain JXY422), FtsW-RFP localizes correctly to midcell and supports normal cell division under our experimental conditions (Extended data Fig. 2a, b, and f). To enable single-molecule detection, we expressed FtsW-RFP ectopically at a low level (plasmid pXY349) in the presence of WT FtsW in BW25113 cells. We tracked single FtsW-RFP molecules (Extended Data Fig.4) at midcell with a $500 \mathrm{~ms}$ exposure time using wide-field fluorescence microscopy. This slow frame rate allowed us to focus on septum-localized FtsW-RFP molecules by effectively filtering out randomly diffusing molecules along the cylindrical part of the cell body. Using a custom-developed unwrapping algorithm, we decomposed two-dimensional (2D) trajectories of individual FtsW-RFP molecules obtained from the curved cell surfaces at midcell to one-dimensional (1D) trajectories along the circumference and long axis of the cell respectively (Extended Data Fig. 5, Methods).

We found that many single FtsW-RFP molecules displayed directional motions at midcell similar to FtsI(12) (Fig. 2a, Supplementary Movie 3). FtsW-RFP molecules also dynamically transitioned between segments of different speeds and directions (Fig. 2b, Extended Data Fig. 6, Supplementary Movie 4-8). These directional motions were not due to sample drifting as such behaviors were almost absent in fixed cells under the same imaging condition $(2.3 \pm 0.4 \%, \mu \pm$ S.E.M., Supplementary Table 6). Some FtsW-RFP molecules displayed confined diffusion and remained largely stationery at the septum (Extended Data Fig. 7a and b, Supplementary Movie S9). Some other FtsW-RFP molecules occasionally entered or left the septum (Movie S6), indicating dynamic exchange of the enzyme between the septum and cell body. Hereafter, we only focus on the dynamics of molecules in the septum where septal cell wall constriction takes place. To quantitatively identify different types of motion of FtsW molecules, we split each trajectory into multiple segments and classified each as stationary or directionally-moving segments based on the total displacement and its associated noise level through statistic means (Fig. 2b, Extended Data Fig. 5e, Methods). Directionally-moving segments were fit to a straight line to extract the directional moving speed $v$ (Fig. $2 \mathrm{a}$ and $\mathrm{b}$ right panels, Extended Data Fig. 5e and 6).

Notably, the speeds of all directionally-moving FtsWRFP molecules showed a much wider distribution (Fig. 2d, top panel, N=315 segments) than the distribution of FtsZ's treadmilling speed(12) (Fig. 2c). The FtsW-RFP's speed distribution was best fit by the sum of two populations, one fast and one slow (Fig. 2d, blue and red curves, Extended Data Fig. 8, Supplementary Table 4 and 9, Methods), distinct from a single fast population as that was seen for FtsZ's treadmilling speed distribution (Fig. 2c, blue curve). The fast-moving population $(62.6 \pm 11.2 \%, \mu \pm$ S.E.M. $)$ of FtsWRFP displayed a mean speed of $31.9 \pm 4.4 \mathrm{~nm} / \mathrm{s},(\mu \pm$ S.E.M., Fig.2d top panel, blue curve and 2e, Supplementary Table 4 ), close to the average FtsZ treadmilling speed we previously measured(12) $(28.0 \pm 1.2 \mathrm{~nm} / \mathrm{s}, \mu \pm$ S.E.M.). The slowmoving population $(37.4 \pm 11.2 \%, \mu \pm$ S.E.M.) of FtsW-RFP molecules had an average speed of $\sim 8 \mathrm{~nm} / \mathrm{s}(8.0 \pm 0.3 \mathrm{~nm} / \mathrm{s}$, Fig. 2d, top panel, red curve, Supplementary Table 4). The existence of, and transition between, the two different types of directional movements could also be directly observed in many individual FtsW-RFP trajectories (Fig. 2b, Extended Data Fig. 6). The essential sPG transpeptidase FtsI, which likely forms a cognate complex with FtsW, exhibits a statistically indistinguishable speed distribution compared to that of FtsW ( $\mathrm{p}=0.32$, K-S test), with similar two directional-moving populations (Extended Data Fig. 9, $46.1 \pm 19.9 \%$ and $31.2 \pm 5.6 \mathrm{~nm} / \mathrm{s}$ for the fast moving population, $53.9 \pm 19.9 \%$ and $9.8 \pm 1.1 \mathrm{~nm} / \mathrm{s}$ for the slow-moving population, $\mu \pm$ S.E.M., $\mathrm{n}=92$ segments, Supplementary Table 5).

To investigate how the two directionally moving populations of FtsW respond to FtsZ's treadmilling dynamics, we performed SMT of FtsW-RFP in five strains with mutations that progressively lower FtsZ's GTPase activity and treadmilling speed (fts ${ }^{E 238 A}, f_{t s} Z^{E 250 A}, f t s Z^{D 269 A}, f t s Z^{G 105 S}$ and $f t s Z^{D 158 A}$, Fig. 2d, Supplementary Table 4). As we previously also observed for FtsI(12), the average speed of all moving FtsW-RFP molecules decreased with the treadmilling speed of FtsZ in these mutant strains (Supplementary Table 4). However, decomposing the speed distribution of FtsW-RFP into two moving populations in each FtsZ GTPase mutant (Fig. 2e, Methods, Extended Data Fig. 8) revealed that only the speed of the fast-moving population is highly correlated with FtsZ's treadmilling speed (Fig. 2e, blue), whereas the slow-moving population maintained a relatively constant speed around 8nm/s independent of FtsZ's treadmilling speed (Fig. 2e, orange, Supplementary Table 4). A similar differential response of FtsI was observed in the $f t s Z^{E 250 A}$ background under the same experimental condition $(19.2 \pm 3.4 \mathrm{~nm} / \mathrm{s}$ and $9.1 \pm 1.8 \mathrm{~nm} / \mathrm{s}$ for the fast and slowmoving populations respectively, $\mu \pm$ S.E.M, $\mathrm{n}=45$ segments, Fig. 2e, cyan and orange markers, Extended Data Fig. 9, Supplementary Table 5). This observation supports the idea that FtsW and FtsI likely move together as part of one sPG synthase complex. The differential responses of the two moving populations of FtsW and FtsI to FtsZ's treadmilling dynamics suggest that the fast-moving population is driven by FtsZ treadmilling dynamics as we showed previously(12), while the slow-moving population is not. Note that a recent study showed that in S. pneumoniae the sPG synthesis complex, FtsW-PBP2x, only exhibited one single, slow-moving population that is FtsZ-independent(17). 
bioRxiv preprint doi: https://doi.org/10.1101/850073; this version posted September 29, 2020. The copyright holder for this preprint (which was not certified by peer review) is the author/funder, who has granted bioRxiv a license to display the preprint in perpetuity. It is made available under aCC-BY-NC-ND 4.0 International license.
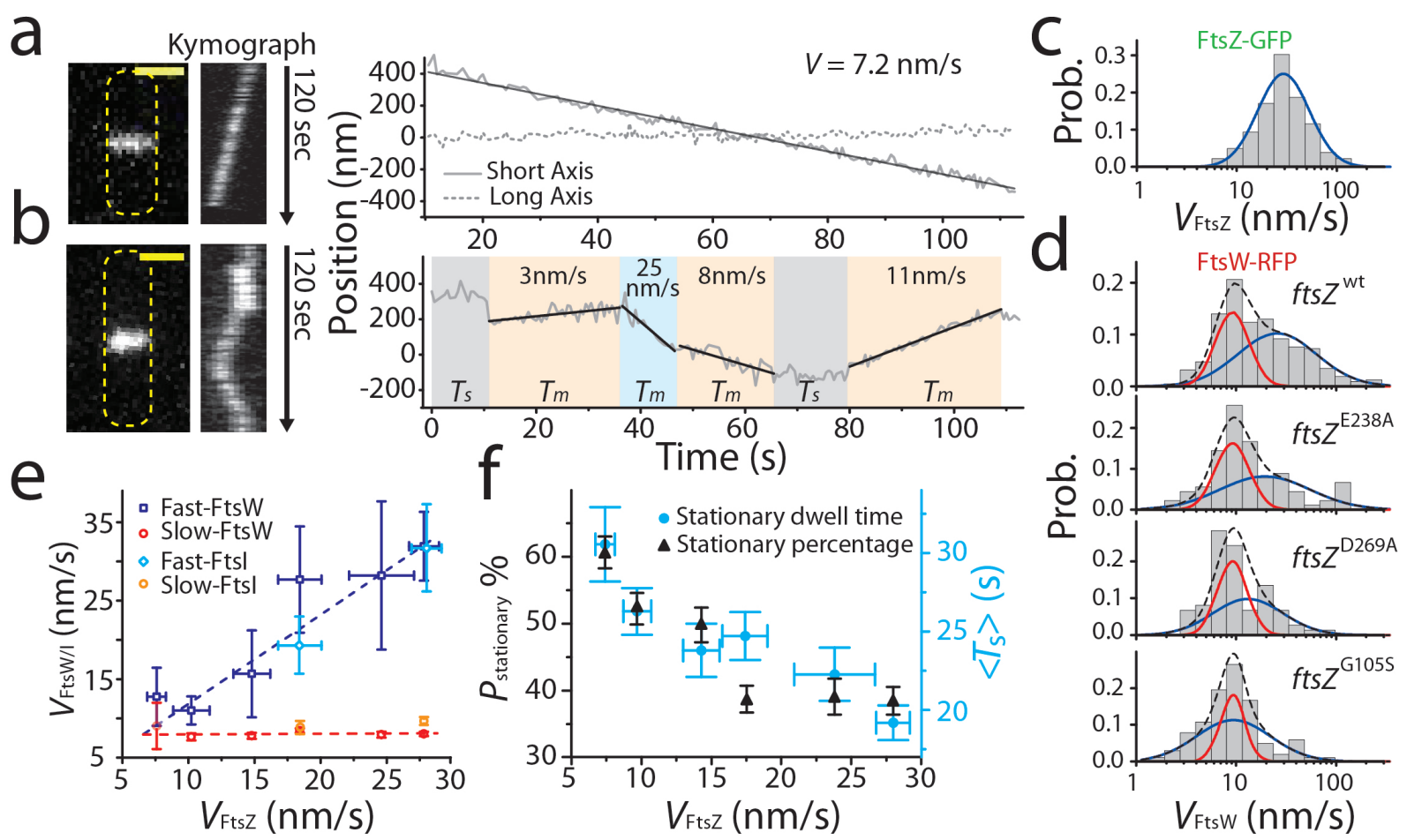

Fig. 2. FtsW exhibits two processive moving populations that are differentially dependent on FtsZ's treadmilling dynamics. a, b: Representative maximum fluorescence intensity projection images (left), kymographs of fluorescence line scans at the septa (middle), and unwrapped one-dimensional (1d) positions of the corresponding FtsW-RFP molecule along the circumference (right, solid gray) and long axis (dot gray) of the cell. (a), one processive moving FtsW-RFP molecule and (b), one moving FtsW-RFP molecule that transitioned between different directions and speeds. The trajectory is segmented into different states of slow-moving (beige), fast-moving (pale blue), and stationary (gray). $T_{m}$ : dwell time of moving segments, $T_{s}$ : dwell time of stationary segments. Scale bars: $1 \mu \mathrm{m}$. c. Treadmilling speed distribution of WT FtsZ adapted from (12) overlaid with the single population fit curve (blue). d. Speed distributions of directional FtsW-RFP molecules in wt and ftsZ GTPase mutant strains overlaid with the double population fit curves (slow-moving population in red, fast-moving population in blue, and the overall fit curve in black dash lines). Note that the red population remained largely unchanged while the blue population gradually moved to slower speeds in FtsZ GTPase mutants. e. Decomposed mean speed of the fast population (blue: FtsW, cyan: Ftsl) is correlated with FtsZ's treadmilling speed while the speed of the slow-moving population (red: FtsW, orange: Ftsl) is independent on FtsZ's treadmilling speed. f. Percentage (dark triangles) and mean dwell time (cyan dots) of stationary FtsW molecules increased with reduced FtsZ treadmilling speed (left to right).

In addition to the two directional moving populations, we investigated how the stationary population of FtsWRFP responded in FtsZ GTPase mutants. Using the mean squared displacement (MSD) analysis, we found that stationary FtsW-RFP molecules in WT FtsZ cells were confined in a region of $\sim 100 \mathrm{~nm}$ in length $(95.5 \pm 6.1 \mathrm{~nm}, \mu \pm$ S.E.M., $\mathrm{N}=179$ segments) with a very small diffusion coefficient ( $\mathrm{D}=0.0007 \pm 0.0002 \mu \mathrm{m}^{2} / \mathrm{s}, \mu \pm$ S.E.M., Extended Data Fig. 7c). We confirmed that this level of confinement was not due to our experimental uncertainty in SMT, as fixed cell SMT showed a much smaller mean square displacement (Extended Data Fig. 7c). Because the confinement length is on par with the average length of FtsZ filaments in the FtsZ-ring (80$160 \mathrm{~nm})(18,19)$, it is possible that some of these stationary FtsW molecules are bound to the middle of FtsZ filaments. Consistent with this possibility, we observed that the average dwell time and percentage of the stationary FtsW-RFP population increased as the treadmilling speed of FtsZ decreased in GTPase mutant strains (Fig. 2f, Supplementary Table 4), in line with the observed increase in the dwell time of FtsZ monomers and FtsZ filament length in FtsZ GTPase mutants in a recent study (20). Interestingly, the average dwell time of stationary FtsW-RFP in wt cells (19.2 \pm 1.1 s, Fig. 2f, Supplementary Table 4) is significantly longer than the lifetime of
FtsZ monomers $(8.1 \pm 0.5 \mathrm{~s})(20)$, indicating that other mechanism(s) may also be at play to maintain this stationary population of FtsW-RFP, as we will further describe below.

Given our results so far that the overall sPG synthesis activity is not affected in FtsZ GTPase mutants in E. coli $(12,21)$, it is likely that the fast-moving population of FtsW and FtsI is inactive in SPG synthesis, but dependent on FtsZ's treadmilling to be transported to different septal positions. We recently have shown that these sPG enzyme molecules could follow FtsZ's fast treadmilling dynamics by continuously tracking the shrinking end of FtsZ filaments through a Brownian Rachet mechanism (22). The FtsZ-independent, slow-moving population, however, may be active in sPG synthesis since their speed is independent of FtsZ. To examine this possibility, we investigated the speed distributions of FtsW-RFP molecules under conditions of altered SPG synthesis activity.

We first examined the movement of FtsW-RFP molecules when its PGTase activity is enhanced or inhibited (Fig. 3a-c). To examine FtsW's motion under enhanced sPG synthesis activity conditions, we tested a superfission (SF) allele of $f t s W\left(f t s W^{E 289 G}\right.$ ) that confers a short-cell phenotype and alleviates the need for FtsN, an otherwise essential positive regulator of SPG synthesis (Extended Data Fig. 
bioRxiv preprint doi: https://doi.org/10.1101/850073; this version posted September 29, 2020. The copyright holder for this preprint (which was not certified by peer review) is the author/funder, who has granted bioRxiv a license to display the preprint in perpetuity. It is made available under aCC-BY-NC-ND 4.0 International license.
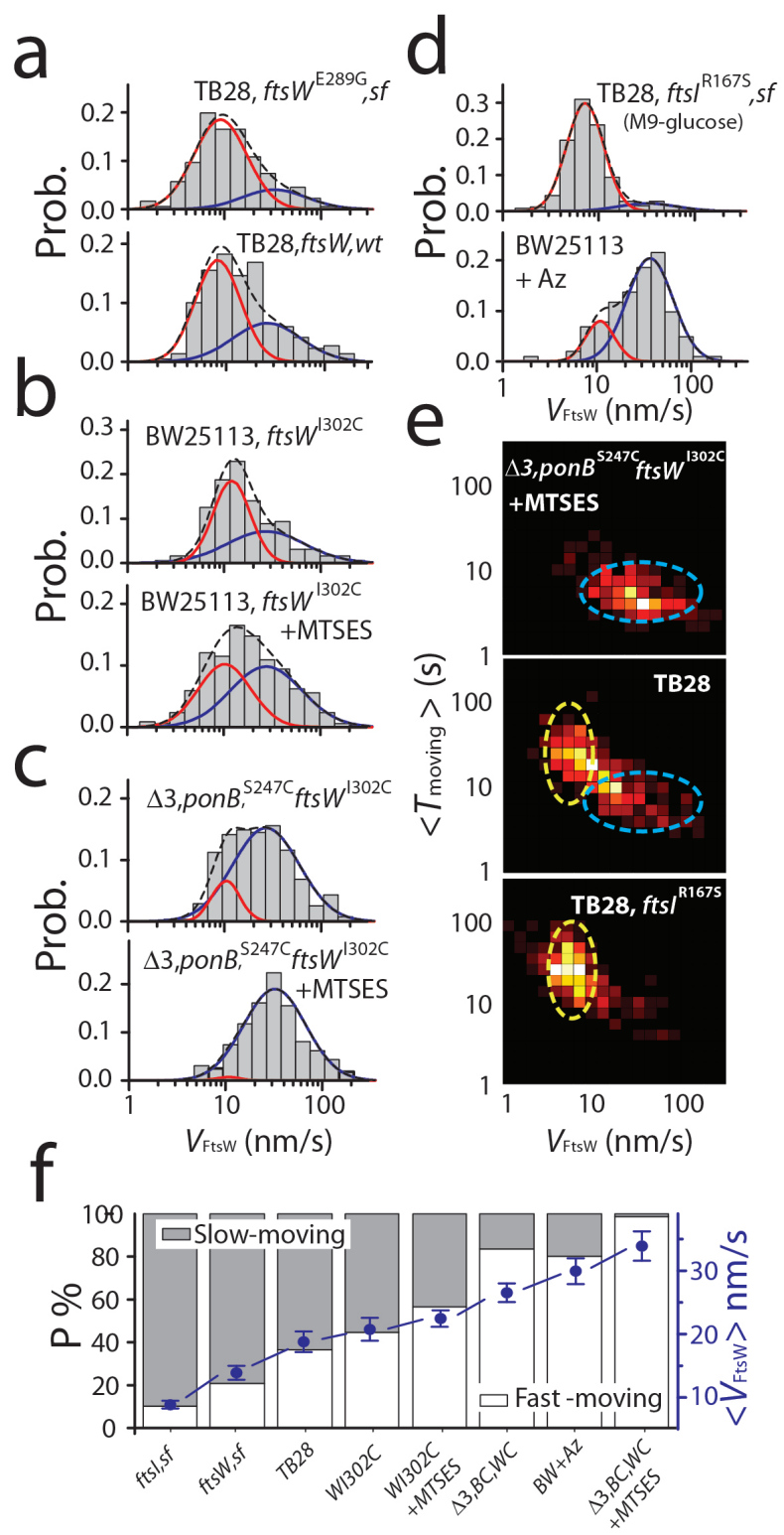

Fig. 3. The slow-moving population of FtsW increases with enhanced sPG synthesis activity and depletes with reduced SPG synthesis activity. a-d. Histograms of directional moving speeds of FtsW-RFP (gray bar) overlaid with two population fitting curves (slow-moving population in red, fast-moving population in blue, and overall fit curve in black dash lines). (a) The superfission variant FtsW ${ }^{E 289 G}$-RFP in the TB28, fts $W^{E 289 G}$ strain background (top) shows increased slow-moving population (red curve) compared to that of wildtype FtsWRFP in the parental TB28 strain background. (b). FtsW ${ }^{E 289 G}$-RFP in JXY559 $\left(f t s W^{E 289 G}\right.$ ) in the absence (top) of MTSES shows increased slow-moving population (red curve) compared to that in the presence of MTSES where FtsW activity is inhibited (bottom). (c). FtsW ${ }^{E 289 G}$-RFP in JXY564 ( $\Delta 3$,ponB ${ }^{S 247 C}$, ftsW $\left.{ }^{I 302 C}\right)$ in the presence of MTSES exhibits nearly completely abolished slow-moving population (bottom) compared to that in the already significantly diminished slow-moving population in the absence of MTSES (top). (d) Wildtype FtsW-RFP in the Ftsl superfission strain TB28, $\mathrm{fts}^{R 167 S}$ background in M9-glucose medium (top) exhibits the most enhanced slow-moving population compared to that in the BW25113 (wt) strain treated with Ftsl-inhibitor Aztreonam. e. 2D-heatmap of the moving speed and dwell time of directional moving FtsW (or FtsW ${ }^{I 302 C}$ )-RFP molecules in normal growth condition (M9-glucose, middle), all PGTase inhibited condition (top), and Ftsl superfission strain (bottom). The slow-moving population with long dwell time (yellow oval) and the fast-moving population with short dwell time (blue oval) are marked with circles as a guide for the eyes. f. Percentage of the slow- (gray bar) and fast- (white bar) moving population under all the conditions in a-d. Average moving speeds under all the conditions are plotted in blue dots.
10). SMT of the SF variant (FtsW ${ }^{E 289 G}$-RFP) in $f t s W^{E 289 G}$ cells again revealed two populations, one fast and one slow as its WT parental strain TB28 (Fig.3a, blue and red curves), but the fast-moving population was reduced from $36.4 \pm 7.6 \%$ to $20.9 \pm 5.6 \%$ (Fig. 3f, Supplementary Table 6). The mean speed of each population did not change significantly compared to that of the WT strain (Supplementary Table 6). Accordingly, the average speed of all directionally moving $\mathrm{FtsW}^{E 289 G}$-RFP molecules in $\mathrm{fts}^{E 289 G}$ cells was significantly slower than that of FtsW-RFP in WT parental TB28 cells $(13.9 \pm 1.1$ vs $18.8 \pm 1.6 \mathrm{~nm} / \mathrm{s}$, Fig. 3f, blue, Supplemental Table 6). These results are consistent with the hypothesis that the slow-moving population of FtsW is coupled to sPG synthesis activity and that the SF FtsW ${ }^{E 289 G}$ variant increased this slow-moving population significantly.

Next, we inhibited partially or completely septal glycan strand polymerization using MTSES on two strains we describe in Fig. 1. We first tracked the movement of $\mathrm{FtsW}^{I 302 C}$-RFP molecules in the BW25113 $\mathrm{fts}^{I 302 C}$ background (JXY559/pAD004) in the absence and presence of MTSES. In the absence of MTSES, we again observed two directionally moving populations of $\mathrm{FtsW}^{I 302 C}$. RFP, one fast at $33.8 \pm 2.7 \mathrm{~nm} / \mathrm{s}(44.6 \pm 7.5 \%)$ and one slow at $10.7 \pm 0.9 \mathrm{~nm} / \mathrm{s}(55.4 \pm 7.5 \%)$. The average speed is at $20.7 \pm 1.8 \mathrm{~nm} / \mathrm{s}(\mu \pm$ s.e.m., $\mathrm{N}=192$ segments, Fig. 3b, top panel, Supplementary Table 6). In the presence of MTSES, the slow-moving population of $\mathrm{FtsW}^{I 302 C}$-RFP was reduced to $43.4 \pm 9.1 \%$ (Fig. $3 b$, bottom panel, $\mu \pm$ s.e.m., $\mathrm{N}=339$ segments, Supplementary Table 6). The average speed increased to $22.5 \pm 1.3 \mathrm{~nm} / \mathrm{s}$ ( $\mu \pm$ s.e.m., Fig. 3f, Supplementary Table $6)$.

Since MTSES specifically blocks FtsW ${ }^{I 302 C}$-dependent sPG synthesis, we reasoned that the remaining slow-moving population of FtsW could be driven by $\mathrm{SPG}$ synthesis from PBP1A, 1B, 1C, and MtgA (Fig. 1). To examine this possibility, we tracked $\mathrm{FtsW}^{I 302 C}$-RFP molecules in the $\Delta 3$,ponB ${ }^{S 247 C}$, fts $W^{I 302 C}$ background (JXY564/pAD004). In this background, even in the absence of MTSES, $\mathrm{FtsW}^{I 302 C}$ already displayed a significantly reduced slowmoving population $(16.5 \pm 6.2 \%, \mu \pm$ s.e.m., $\mathrm{N}=276$ segments, Fig. 3c) accompanied by an increased fast moving population compared to WT TB28 cells $(63.6 \pm 7.6 \%, \mu \pm$ s.e.m., $\mathrm{N}=219$ segments, Fig. 3a, bottom panel). These changes are consistent with the reduced alkyne-NAM incorporation in this strain background (Extended Data Figure. 3), suggesting that the decrease of the overall PGTase activity indeed depletes the slow-moving population. The average speed also increased to $26.3 \pm 1.5 \mathrm{~nm} / \mathrm{s}$. Further abolishing all PGTase activity by $0.1 \mathrm{mM}$ MTSES ( $\sim 99 \%$ reduction, Supplementary Table 3) caused a near complete depletion of the slow-moving population to $1.2 \pm 2.7 \%$, (Fig. $3 \mathrm{c}$ bottom panel and $\mathrm{f}$ ), with the fast-moving population speed remaining at $32.1 \pm 1.5 \mathrm{~nm} / \mathrm{s}$ (Supplementary Table 6).

To ensure that the depletion of slow-moving FtsW molecules is not caused by altered FtsZ treadmilling dynamics under these conditions, we measured FtsZ dynamics in those strains in the presence or absence of MTSES. 
bioRxiv preprint doi: https://doi.org/10.1101/850073; this version posted September 29, 2020. The copyright holder for this preprint (which was not certified by peer review) is the author/funder, who has granted bioRxiv a license to display the preprint in perpetuity. It is made available under aCC-BY-NC-ND 4.0 International license.

FtsZ's treadmilling speed distribution remained unchanged (Extended Data Fig. 11b, Supplementary Table 6), supporting the hypothesis that the slow-moving population of FtsW is related to sPG synthesis activity rather than to FtsZ treadmilling.

To further verify that the slow-moving population of FtsW-RFP is related to active sPG synthesis, we monitored the movement of FtsW-RFP in cells expressing a superfission variant of FtsI ( $\mathrm{FtsI}^{R 167 S}$ ). FtsI ${ }^{R 167 S}$ is similar to $\mathrm{FtsW}^{E 289 G}$ in alleviating the essentiality of FtsN but does so only partially (Extended Data Fig. 12, Table S7). In $\mathrm{ftsI}^{R 167 S}$ cells, a major population of FtsW-RFP $(90.7 \pm 2.1 \%$,$) moved slowly at 6.4 \pm 0.2 \mathrm{~nm} / \mathrm{s}$ and a minor population moved fast at $31.6 \pm 2.0 \mathrm{~nm} / \mathrm{s}(9.3 \pm 2.1 \%, \mathrm{~N}=255$ segments, Fig. 3d, top panel, Supplementary Table 6). The average speed is drastically reduced to $8.8 \pm 0.6 \mathrm{~nm} / \mathrm{s}$ (Fig. $3 \mathrm{f}$, Supplementary Table 6). When we applied FtsI-specific $\beta$ lactam Aztreonam to inhibit its transpeptidase (TPase) activity $(1 \mu \mathrm{g} / \mathrm{mL}, 30 \mathrm{~min})(23)$ in WT BW25113 cells, we observed that the slow-moving population of FtsW-RFP was depleted from $37.4 \pm 11.2 \%$ to $19.8 \pm 7.7 \%$ (Fig. 3d, red curve), whereas the fast-moving population increased with a similar speed distribution as FtsZ's treadmilling dynamics (Fig. 3d, blue curve, Extended Data Fig. 11b). The average speed of FtsW-RFP became $29.9 \pm 2.0 \mathrm{~nm} / \mathrm{s}(\mathrm{N}=102$ segments). These results not only support the hypothesis that the slow-moving population of FtsW depends on active SPG synthesis, but also indicate that the crosslinking of sPG by FtsI's TPase activity is equally important as the polymerization of glycan strands to drive the progressive movement of the slow population of the sPG synthase.

Most interestingly, we found that the two moving populations of FtsW are also separated in the time domain. We measured the dwell time that individual FtsW-RFP molecules spent at a constant speed (as depicted in Fig. $2 \mathrm{~b}$ ) and plotted the corresponding two-dimensional (2D)histogram of the moving speed and dwell time in Fig.3e. We found that the slow-moving population $(\sim 8 \mathrm{~nm} / \mathrm{s})$ is associated with a relative long dwell time $(\sim 25 \mathrm{sec}$, yellow circle in Fig. 3e middle panel) and the fast-moving population $(\sim 30 \mathrm{~nm} / \mathrm{s})$ with a significantly shorter one ( $6 \mathrm{sec}$, blue circle in Fig. 3e). In the sPG synthesis inhibited $\left(\triangle 3\right.$, ponB $^{S 247 C}$, fts $^{I 302 C}+$ MTSES $)$ condition, the fastmoving, short-lived population $(5.8 \pm 3.2 \mathrm{sec}, \mu \pm$ s.d.) became dominant (Fig. 3e, top panel, Extended Data Fig. 13), while the slow-moving, long-lived population $(25.3 \pm 15.6$ sec, $\mu \pm$ s.d.) dominated in the superfission $f t s I^{R 167 S}$ background (Fig. 3e bottom panel, Extended Data Fig. 13). These observations further confirmed that the two moving populations of FtsW-RFP molecules are distinct from each other.

One factor controlling the SPG synthesis rate is the availability of cell wall precursors. In E. coli under balanced growth, the cellular level of precursors limits cell growth and cell wall constriction rates $(24,25)$. We reasoned that if the slow-moving population of FtsW were indeed driven by sPG synthesis activity, its size and even its speed might be modulated by the level of available PG precursors. To
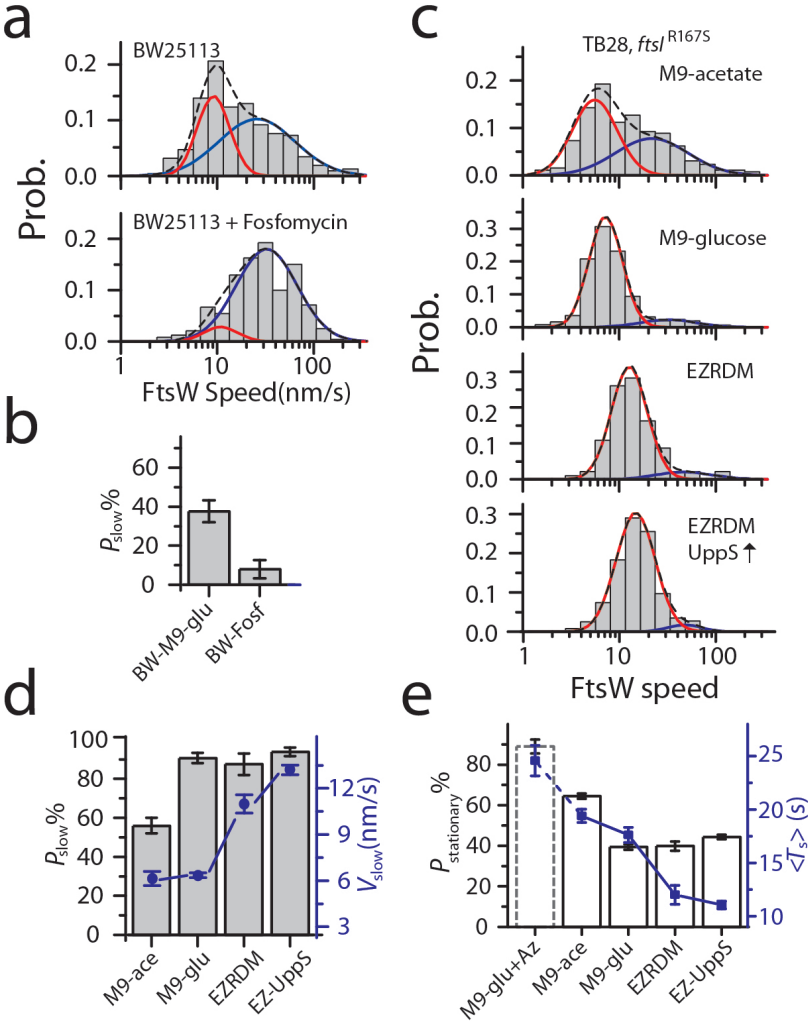

Fig. 4. Enhanced growth conditions promote the slow-moving population of FtsW. a, c. Histograms of directional moving speeds of FtsW-RFP (gray bar) overlaid with slow-(red) and fast-(blue) moving population fit curves, and the overall fit curve (black dash). (a) FtsW-RFP in BW25113 cells in M9-glucose medium (top, replot from Fig. 2d) and treated with Fosfomycin to deplete the lipid II precursor pool (bottom). (b) The slow-moving population of FtsW-RFP decreases upon depletion of lipidll (Fosfomycin treatment). c. FtsW-RFP in FtsI superfission strain TB28, fts ${ }^{R 167 S}$ grown in M9-acetate, M9-glucose, EZRDM, or in EZRDM medium and with UppS overproduction (top to bottom). d. The percentage (gray bar) and average speed (blue dot) of the slow-moving population increases in media with enhanced growth conditions. e. Percentage (white bars) of stationary FtsWRFP molecules decreases and reaches a plateau with enhanced growth conditions whereas and average dwell time (blue squares) continues to decrease. BW25113 wt cells treated with Aztreonam treatment (dashed bar, M9-glu+Az) is shown as a comparison. The corresponding constriction rates for all the growth conditions are listed in Supplementary Table 8.

test this possibility, we first depleted precursor Lipid II in WT BW25113 cells by blocking the essential enzyme MurA using Fosfomycin(26). We found that in cells treated with Fosfomycin $(50 \mu \mathrm{g} / \mathrm{ml}, 30 \mathrm{~min})$, the slow-moving population of FtsW was significantly diminished to $\sim 8 \%(7.9 \pm 9.3 \%$, $\mathrm{N}=239$ segments, Fig. 4a and b, Supplementary Table 6) while the fast-moving population dominated with unchanged moving speed.

Next, we used M9 minimal growth medium containing acetate or glucose as the carbon source or EZ rich defined medium (EZRDM) to vary the available levels of PG precursors in the superfission fts ${ }^{R 167 S}$ background (strain PM6). We measured the corresponding cell wall constriction rates using an mNeonGreen-ZapA fusion protein to confirm that cells growing in these different media exhibited different sPG synthesis activities. Indeed, the constriction rate increased from $4.9 \pm 1.1 \mathrm{~nm} / \mathrm{min}(\mathrm{N}=5$ cells) in M9-acetate, to $17.2 \pm 1 \mathrm{~nm} / \mathrm{min}(\mathrm{N}=15$ cells $)$ in M9-glucose, and finally to 
$37.9 \pm 2.0 \mathrm{~nm} / \mathrm{min}(\mathrm{N}=16$ cells) in EZRDM medium (Method, Extended Data Fig. 14, Supplementary Table 8). We then monitored the motion of FtsW-RFP under these different growth conditions. As shown in Fig. 4c, the fast-moving population of FtsW, originally diminished in the M9-glucose medium in the superfission variant background (Fig. 3d, top panel, replotted in the second panel of Fig. 4c for a comparison), re-appeared under the M9-acetate growth condition ( $44.4 \pm 4.8 \%, \mathrm{~N}=260$ segments, Fig. $4 \mathrm{c}$ top panel). This result, together with our observation using Fosfomycin, strongly supports our hypothesis that the percentage of slowmoving FtsW molecules is governed by sPG synthesis activity, here determined by the level of cell wall precursors.

In rich growth medium (EZRDM), the percentage of slow-moving FtsW-RFP molecules in PM6 (ftsI ${ }^{R 167 S}$ ) cells stayed around $90 \%(87.5 \pm 5.5 \%$, Fig. 4d), similar to that in M9-glucose. However, the average speed of the slow-moving population of FtsW-RFP accelerated to $11.0 \pm 0.6 \mathrm{~nm} / \mathrm{s}(\mu \pm$ s.e.m., $\mathrm{N}=92$ segments, Fig. 4c second and third panel, $4 \mathrm{~d}$, blue) in EZRDM, $\sim 72 \%$ higher than in M9-glucose $(6.4 \pm 0.2 \mathrm{~nm} / \mathrm{s})$. This faster speed of FtsW-RFP is still significantly slower than that of FtsZ's treadmilling speed under the same growth condition (Extended Data Fig. 11c, Supplementary Table 6), consistent with the expectation that this population is not coupled to FtsZ's treadmilling dynamics. Note that growth of wt BW25113 cells in EZDRM also led to an increased speed of the slow-moving population of FtsW-RFP $(11.1 \pm 0.2 \mathrm{~nm} / \mathrm{s}, \mu \pm$ s.e.m., $\mathrm{n}=910$ segments, Supplementary Table 6), indicating that this medium effect is not specific to the superfission FtsI variant in PM6 cells.

Finally, to further upregulate Lipid II precursor levels in PM6 (ftsI ${ }^{R 167 S}$ ) cells, we grew these cells in EZDRM and induced the expression of the undecaprenyl pyrophosphate synthetase UppS from a plasmid(9). Under this condition, we observed a further increase in the slow-moving speed of FtsW-RFP to $13.2 \pm 0.3 \mathrm{~nm} / \mathrm{s}$ ( $\mu \pm$ s.e.m., $\mathrm{N}=513 \mathrm{seg}$ ments, Fig. 4c bottom panel and 4d, blue). Taken together, these results strongly suggest that the percentage of the slowmoving population reflects the relative amount of active FtsW molecules engaged in SPG synthesis, and its speed likely reflects the in vivo SPG polymerization rate at the individual enzyme molecule level.

In the above experiments, we also observed changes in the percentages and dwell times of the stationary population of FtsW-RFP (Fig. 4e). The percentage of stationary molecules decreased as the expected cell wall precursor levels increased and reached a plateau at $~ 40 \%$ under the M9glucose, EZRDM and UppS-overexpression conditions (Fig. $4 \mathrm{e}$, white bars). The average dwell time of stationary FtsWRFP molecules, however, continued to decrease to $\sim 10$ s (Fig. $4 \mathrm{e}$, blue squares), approaching the mean lifetime of FtsZ monomers in the Z-ring (8.1 sec)(20).

These results, together with those from the experiments of FtsZ GTPase mutants (Fig. 2f), suggest that at least two subpopulations of stationary FtsW molecules may exist. One likely represents molecules bound to internal subunits of FtsZ filaments, and their dwell times and abundance are deter- mined by the length and treadmilling speed of FtsZ filaments (Fig. 2f). The other subpopulation may represent FtsW molecules that are poised at sPG synthesis sites waiting for lipid II to become available. This possibility is supported by our observations that the percentage and mean dwell time of this subpopulation indeed shrank as the level of cell wall precursors increased in rich medium (M9-glucose and EZRDM) or with upregulated UppS expression (Fig. 4d, Extended Data Fig. 7d and e, Supplementary Table 6).

We further reasoned that Aztreonam treatment could substantially enhance the stationary population. Under this condition FtsW engages in a futile sPG synthesis cycle in which it continues to polymerize glycan strands, but these are blocked from being crosslinked by FtsI and subsequently degraded(27). Therefore, the lack of FtsI's crosslinking activity may prevent directional movement of the enzyme while the continuous polymerization activity of FtsW prevents its dissociation from glycan strands. Consistent with this expectation, the dwell time and percentage of the stationary population of FtsW-RFP in Aztreonam-treated cells increased to $24.6 \pm 1.4 \mathrm{sec}$ and $88.9 \pm 3.4 \%$ respectively ( $\mathrm{n}=347$ segments, Fig. 4e, Supplementary Table 6). Furthermore, the MSD curve of stationary FtsW-RFP molecules became indistinguishable from that of the fixed sample (Extended Data Fig. $7 \mathrm{c}$, green), suggesting they also become confined to a smaller region after Aztreonam treatment. Note that previously a stationary, inactive, and PG-bound state has been reported for $E$. coli $\mathrm{PBP} 2$, the counterpart of FtsI in the cell elongation machinery(28).

Our results so far demonstrated that two processive moving populations of FtsW exist in vivo. The fast-moving population is driven by FtsZ's treadmilling dynamics but inactive in sPG synthesis, whereas the slow-moving population is most likely active and driven by SPG synthesis. The presence of active and inactive FtsW populations raises an interesting question: Do division proteins involved in controlling the initiation and progression of cell wall constriction also determine the partitioning of these two populations? Previous studies in E. coli have shown that when FtsW and FtsI are first recruited to the division site by a complex of the FtsB, FtsL and FtsQ proteins (FtsQLB), they are kept in an inactive state until FtsN, the last essential division protein to accumulate at the site, activates the sPG synthesis complex $(29,30)$. Thus, FtsN may play an important role in triggering the transition of FtsW from the fast-moving (FtsZ-dependent) to slow-moving (sPG synthesis-dependent) state.

To test this hypothesis, we used an FtsN-depletion strain (EC1908) (31) wherein the chromosomal fts $N$ gene is controlled by the $\operatorname{araBAD}$ regulatory region. This strain grows and divides normally in the presence of $0.2 \%$ of arabinose (Extended Data Fig. 15). After overnight growth $(\sim 15$ hours) in M9-glucose medium without arabinose, the average cellular FtsN level was depleted to $\sim 44 \%$ of that in WT cells (Extended Data Fig. 15) and many cells grew into long filaments with shallow constrictions (Fig.5a, bottom panel). We tracked FtsW-RFP molecules at shallow constriction sites in these filamentous cells. As expected, the slow- 


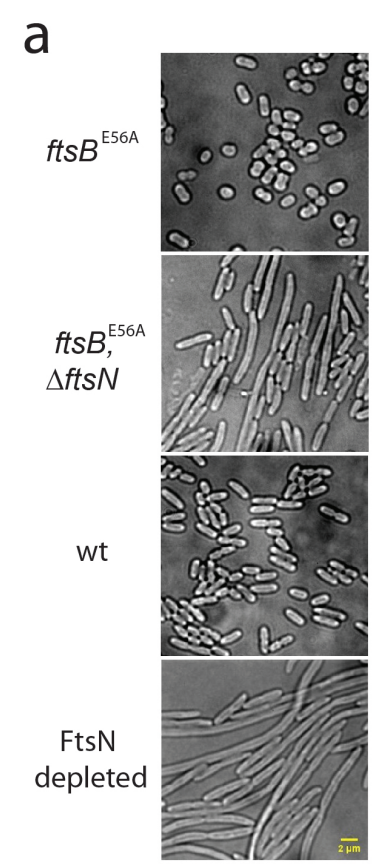

b

C

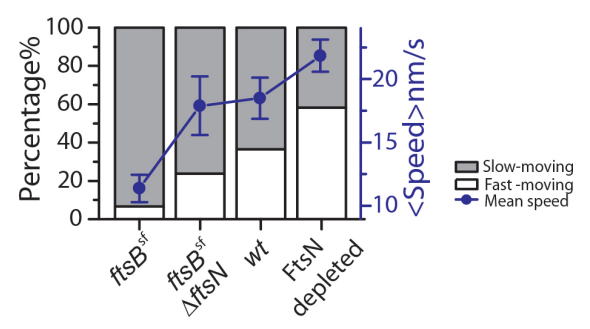

Fig. 5. FtsN plays an important role in promoting the slow-moving population of FtsW. a. Bright-field images of superfission ftsB ${ }^{E 56 A}$ (BL167), ftsB ${ }^{E 56 A} \Delta$ ftsN (BL173), WT (TB28), and FtsN-depleted cells (EC1908). Scale bar: $2 \mu \mathrm{m}$. b. Histograms of directional moving speeds of FtsW-RFP (gray bar) overlaid with slow(red) and fast-moving (blue) population fit curves, and the overall fit curve (black dash) in cells corresponding to a on the left (in M9-glucose medium). c. Average FtsW-RFP moving speeds (blue dots) and percentage of the slow-(gray bar) and fast-(white bar) moving populations under the conditions in $a-b$.

moving population of FtsW-RFP was reduced significantly from $63.6 \pm 7.6 \%$ to $41.7 \pm 14.7 \%$ (Fig. $5 \mathrm{~b}$, third and bottom panels, orange curves, Supplementary Table 6), and the increased fast-moving population of FtsW-RFP molecules moved at an average speed of $30.1 \pm 3.9 \mathrm{~nm} / \mathrm{s}(\mu \pm$ s.e.m., $\mathrm{N}=398$ segments), similar to that of FtsZ treadmilling. This result suggests that FtsN plays a role in promoting the slowmoving, or active, population of FtsW.

Next, to assess the effects of FtsN on FtsW dynamics under conditions were FtsN is no longer essential, we tracked the movements of FtsW-RFP in a fts $B^{E 56 A}$ superfission strain (BL167) that still produces FtsN, and also in strain BL173 (ftsB $B^{E 56 A}, \Delta f t s N$ ) that lacks FtsN completely. The $f t s B^{E 56 A}$ superfission allele causes cells to initiate sPG synthesis earlier in the division cycle than normal, leading to a small-cell phenotype (29) (Fig. 5a, top panel). While it also allows cells to grow and divide in the complete absence of FtsN, fts $B^{E 56 A}, \Delta f t s N$ cells divide less efficiently than wt and are modestly elongated (29) (Fig. 5a, sec- ond panel, Supplementary Table 7). In $f t s B^{E 56 A}$ cells, we observed a significant increase in the slow-moving population of FtsW (Fig. 5b, top panel, orange curves); the fastmoving, FtsZ-dependent population of FtsW-RFP was essentially abolished, and nearly all directional moving FtsW-RFP molecules moved at an average speed of $\sim 9 \mathrm{~nm} / \mathrm{s}(9.3 \pm 0.8$ $\mathrm{nm}, 93.3 \pm 3.7 \%, \mathrm{~N}=176$ segments, Fig. $5 \mathrm{~b}$ and c, orange, Supplementary Table 6). Most interestingly and as expected, in $f t s B^{E 56 A}, \Delta f t s N$ cells where FtsN is absent, the slow-moving population of FtsW-RFP was reduced while the fast-moving population recovered to a level close to that in WT cells $(23.8 \pm 4.4 \%, \mathrm{~N}=144$ segments, Fig. 5b second panel, Supplementary Table 6). These results indicate that even though FtsN is no longer essential in the superfission $f t s B^{E 56 A}$ background, it still contributes to the transitioning of FtsW from the fast-moving, FtsZ-dependent state to the slow-moving, sPG synthesis-dependent mode.

\section{Discussion}

Taken together, our data support a two-track model (Fig.6), in which directionally moving FtsW and FtsI, and possibly other sPG remodeling enzymes and regulators as well, occupy at least two "tracks" within the septum: a fast "Ztrack" representing inactive molecules end-trailing treadmilling FtsZ polymers(22), and a slow "sPG-track" representing active molecules that exited the Z-track to produce sPG. Based on the speed of slow-moving FtsW and FtsI, the sPG polymerization rate in live E. coli cells is likely $\sim 6$ to 14 disaccharides per second (one disaccharide is $\sim 1 \mathrm{~nm}$ (32)), on par with what we estimated from previous biochemical labeling experiments (33) (Supplementary). Stationary FtsWI molecules are likely bound to the middle of FtsZ filaments or trapped at SPG synthesis sites waiting for available lipid II or other factors to initiate/continue sPG synthesis, or to depart the sPG-track to engage FtsZ again. FtsN promotes the release of inactive sPG synthase from treadmilling FtsZ polymers to pursue the sPG-track for active synthesis. In this scenario, FtsWI may associate with regulators of sPG synthesis activity such as FtsN and FtsQLB on either or both tracks, and switch between active and inactive states based on appropriate input by the regulatory proteins. Future SMT studies on the dynamics of the FtsQLB and FtsN proteins should help elucidate how they control FtsWI activity and cell constriction in molecular details. The two-track model integrates spatial information into the regulation of sPG synthesis and suggests a novel mechanism for the spatiotemporal coordination of bacterial cell wall constriction.

\section{Acknowledgements}

The authors thank lab members in the Xiao and de Boer labs for helpful discussions and technical assistance, Dr. G. Hauk for sharing plasmids and the CRISPR-Cas $9 / \lambda$-red recombineering cloning method, Dr. D. S. Weiss for strain EC1908, plasmid pDSW406, anti-FtsN serum, and helpful suggestions on FtsW immunoblotting, Dr. T. Berhardt for strain HC532 and plasmid pHC808, Dr. C. Hale for plasmid pCH650, 
bioRxiv preprint doi: https://doi.org/10.1101/850073; this version posted September 29, 2020. The copyright holder for this preprint (which was not certified by peer review) is the author/funder, who has granted bioRxiv a license to display the preprint in perpetuity. It is made available under aCC-BY-NC-ND 4.0 International license.
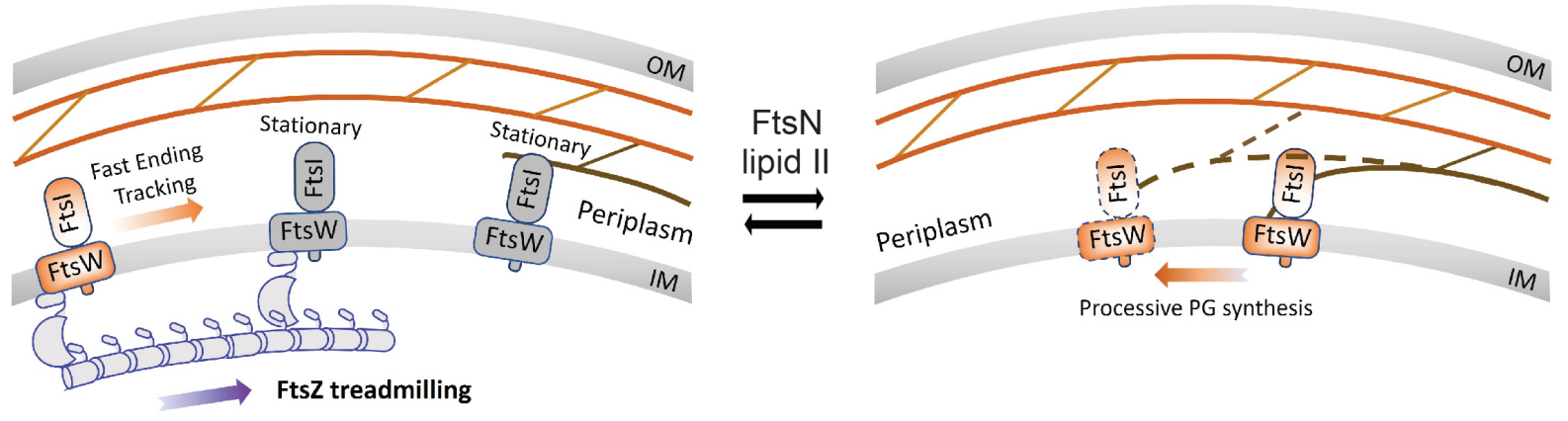

Fig. 6. A two-track model integrating spatial information into the regulation of SPG synthase activity. Inactive synthase FtsWI complex follows the treadmilling FtsZ filament (Z-track, left) and is transported to different locations along the septum. Active FtsWI complex engages in processive septal cell wall synthesis along the sPG track (right). FtsN and the available level of cell wall synthesis precursor Lipid II play important roles in promoting the release of inactive FtsWI from the Z-track to pursue sPG synthesis on the SPG-track. Stationary FtsWI complexes (grey molecules, left panel) likely include those bound to internal subunits of FtsZ filaments until the shrinking end approaches, and those bound at SPG synthesis sites waiting for factors (e.g. lipid II substrate or the activator FtsN) to start or continue sPG synthesis.

Dr. E. Goley for help on cell growth measurement, and Dr. R. Tsien for the TagRFP-T construct. This work was supported by NIH GM57059 (to P.d.B), NIH R01 GM086447 (to J.X.), GM125656 (subcontract to J. X.), NSF EAGER Award MCB-1019000 (to J.X.), and a Hamilton Smith Innovative Research Award (to J.X .)

\section{Reference}

1. Hongbaek Cho, Carl N. Wivagg, Mrinal Kapoor, Zachary Barry, Patricia D.A. Rohs, Hyunsuk Suh, Jarrod A. Marto, Ethan C. Garner, and Thomas G. Bernhardt. Bacterial cell wall biogenesis is mediated by SEDS and PBP polymerase families functioning semiAutonomously. Nature Microbiology, 1, 2016. ISSN 20585276. doi: 10.1038/nmicrobiol. 2016.172.

2. Emily K. Butler, Wee Boon Tan, Hildy Joseph, and Natividad Ruiz. Charge requirements of lipid II flippase activity in Escherichia coli. Journal of Bacteriology, 196(23):4111-4119, 2014. ISSN 10985530. doi: 10.1128/JB.02172-14.

3. Megan Sjodt, Kelly Brock, Genevieve Dobihal, Patricia D.A. Rohs, Anna G. Green, Thomas A. Hopf, Alexander J. Meeske, Veerasak Srisuknimit, Daniel Kahne, Suzanne Walker, Debora S. Marks, Thomas G. Bernhardt, David Z. Rudner, and Andrew C. Kruse. Structure of the peptidoglycan polymerase RodA resolved by evolutionary coupling analysis. Nature, 556(7699):118-121, 2018. ISSN 14764687. doi: 10.1038/nature25985.

4. Arthur Karlin and Myles H. Akabas. Substituted-cysteine accessibility method. Methods in Enzymology, 293:123-145, 1998. ISSN 00766879. doi: 10.1016/S0076-6879(98)93011-7.

5. Alexander J. Meeske, Eammon P. Riley, William P. Robins, Tsuyoshi Uehara, John J. Mekalanos, Daniel Kahne, Suzanne Walker, Andrew C. Kruse, Thomas G. Bernhardt, and David Z. Rudner. SEDS proteins are a widespread family of bacterial cell wall polymerases. Nature, 537(7622):634-638, 2016. ISSN 14764687. doi: 10.1038/nature19331.

6. Erkin Kuru, Srinivas Tekkam, Edward Hall, Yves V. Brun, and Michael S. Van Nieuwenhze. Synthesis of fluorescent D-amino acids and their use for probing peptidoglycan synthesis and bacterial growth in situ. Nature Protocols, 10(1):33-52, 2015. ISSN 17502799. doi: 10.1038/nprot.2014.197.

7. Hai Liang, Kristen E. DeMeester, Ching Wen Hou, Michelle A. Parent, Jeffrey L. Caplan, and Catherine L. Grimes. Metabolic labelling of the carbohydrate core in bacterial peptidoglycan and its applications. Nature Communications, 8:1-11, 2017. ISSN 20411723. doi: 10.1038/ ncomms 15015.

8. Hartmuth C. Kolb, M. G. Finn, and K. Barry Sharpless. Click Chemistry: Diverse Chemical Function from a Few Good Reactions, 2001. ISSN 14337851.

9. Lok To Sham, Emily K. Butler, Matthew D. Lebar, Daniel Kahne, Thomas G. Bernhardt, and Natividad Ruiz. MurJ is the flippase of lipid-linked precursors for peptidoglycan biogenesis. Science, 345(6193):220-222, 2014. ISSN 10959203. doi: 10.1126/science.1254522.

10. Alexander J.F. Egan and Waldemar Vollmer. The physiology of bacterial cell division. Annals of the New York Academy of Sciences, 1277(1):8-28, 2013. ISSN 17496632. doi: 10.1111/ j.1749-6632.2012.06818.x.

11. Atsushi Taguchi, Michael A. Welsh, Lindsey S. Marmont, Wonsik Lee, Megan Sjodt, Andrew C. Kruse, Daniel Kahne, Thomas G. Bernhardt, and Suzanne Walker. FtsW is a peptidoglycan polymerase that is functional only in complex with its cognate penicillinbinding protein. Nature Microbiology, 4(4):587-594, 2019. ISSN 20585276. doi: 10.1038/ s41564-018-0345-x.

12. X. Yang, Z. Lyu, A. Miguel, R. McQuillen, K.C. Huang, and J. Xiao. GTPase activity-coupled treadmilling of the bacterial tubulin FtsZ organizes septal cell wall synthesis. Science, 355 (6326):744-747, 2017. ISSN 10959203. doi: 10.1126/science.aak9995.

13. A. W. Bisson-Filho, Y. P. Hsu, G. R. Squyres, E. Kuru, F. Wu, C. Jukes, Y. Sun, C. Dekker, S. Holden, M. S. VanNieuwenhze, Y. V. Brun, and E. C. Garner. Erratum: Treadmilling by FtsZ filaments drives peptidoglycan synthesis and bacterial cell division (Science DOI: 10.1126/science.aak9973). Science, 367(6475):6-10, 2020. ISSN 10959203. doi: 10.1126/ science.aba6311.

14. Sophie Leclercq, Adeline Derouaux, Samir Olatunji, Claudine Fraipont, Alexander J.F. Egan, Waldemar Vollmer, Eefjan Breukink, and Mohammed Terrak. Interplay between Penicillin- binding proteins and SEDS proteins promotes bacterial cell wall synthesis. Scientific Reports, 7(November 2016):1-13, 2017. ISSN 20452322. doi: 10.1038/srep43306.

15. Claudine Fraipont, Svetlana Alexeeva, Benoît Wolf, Renévan Der Ploeg, Marie Schloesser, Tanneke Den Blaauwen, and Martine Nguyen-Distèche. The integral membrane FtsW protein and peptidoglycan synthase PBP3 form a subcomplex in Escherichia coli. Microbiology, 157(1):251-259, 2011. ISSN 13500872. doi: 10.1099/mic.0.040071-0.

16. Sergey Ovchinnikov, Lisa Kinch, Hahnbeom Park, Yuxing Liao, Jimin Pei, David E. Kim, Hetunandan Kamisetty, Nick V. Grishin, and David Baker. Large-scale determination of previously unsolved protein structures using evolutionary information. eLife, 4(September): 1-25, 2015. ISSN 2050084X. doi: 10.7554/eLife.09248.

17. Amilcar J. Perez, Yann Cesbron, Sidney L. Shaw, Jesus Bazan Villicana, Ho Ching T. Tsui, Michael J. Boersma, Ziyun A. Ye, Yanina Tovpeko, Cees Dekker, Seamus Holden, and Malcolm E. Winkler. Movement dynamics of divisome proteins and PBP2x: FtsW in cells of Streptococcus pneumoniae. Proceedings of the National Academy of Sciences of the United States of America, 116(8):3211-3220, 2019. ISSN 10916490. doi: 10.1073/pnas. 1816018116.

18. Bill Söderström, Helena Chan, Patrick J. Shilling, Ulf Skoglund, and Daniel O. Daley. Spatial separation of FtsZ and FtsN during cell division. Molecular Microbiology, 107(3):387-401, 2018. ISSN 13652958. doi: $10.1111 / \mathrm{mmi} .13888$.

19. Zhuo Li, Michael J. Trimble, Yves V. Brun, and Grant J. Jensen. The structure of FtsZ filaments in vivo suggests a force-generating role in cell division. EMBO Journal, 26(22): 4694-4708, 2007. ISSN 02614189. doi: 10.1038/sj.emboj.7601895.

20. Georgia R Squyres, Matthew J Holmes, Sarah R Barger, Betheney R Pennycook, Joel Ryan, Victoria $T$ Yan, and Ethan $C$ Garner. Dynamics of bacterial cell division: $Z$ ring condensation is essential for cytokinesis. bioRxiv, page 2020.06.30.180737, 2020. doi: 10.1101/2020.06.30.180737.

21. Carla Coltharp, Jackson Buss, Trevor M. Plumer, and Jie Xiao. Defining the rate-limiting processes of bacterial cytokinesis. Proceedings of the National Academy of Sciences of the United States of America, 113(8):E1044-E1053, 2016. ISSN 10916490. doi: 10.1073/ pnas. 1514296113.

22. Joshua McCausland, Xinxing Yang, Georgia Squyres, Zhixin Lyu, Kevin Bruce, Melissa Lamanna, Bill Söderström, Ethan Clark Garner, Malcolm Winkler, Jie Xiao, and Jian Liu. Treadmilling FtsZ polymers drive the directional movement of sPG-synthesis enzymes via a Brownian ratchet mechanism. pages 1-38, 2019. doi: 10.1101/857813.

23. Ozden Kocaoglu and Erin E. Carlson. Profiling of $\beta$-lactam selectivity for penicillin-binding proteins in Escherichia coli strain DC2. Antimicrobial Agents and Chemotherapy, 59(5): 2785-2790, 2015. ISSN 10986596. doi: 10.1128/AAC.04552-14.

24. Timothy K. Lee, Carolina Tropini, Jen Hsin, Samantha M. Desmarais, Tristan S. Ursell, Enhao Gong, Zemer Gitai, Russell D. Monds, and Kerwyn Casey Huang. A dynamically assembled cell wall synthesis machinery buffers cell growth. Proceedings of the National Academy of Sciences of the United States of America, 111(12):4554-4559, 2014. ISSN 10916490. doi: 10.1073/pnas.1313826111.

25. Enrique Rojas, Julie A. Theriot, and Kerwyn Casey Huang. Response of Escherichia coli growth rate to osmotic shock. Proceedings of the National Academy of Sciences of the United States of America, 111(21):7807-7812, 2014. ISSN 10916490. doi: 10.1073/pnas. 1402591111.

26. Lynn L. Silver. Fosfomycin: Mechanism and resistance. Cold Spring Harbor Perspectives in Medicine, 7(2):1-11, 2017. ISSN 21571422. doi: 10.1101/cshperspect.a025262.

27. Hongbaek Cho, Tsuyoshi Uehara, and Thomas G. Bernhardt. Beta-lactam antibiotics induce a lethal malfunctioning of the bacterial cell wall synthesis machinery. Cell, 159(6): 1300-1311, 2014. ISSN 10974172. doi: 10.1016/j.cell.2014.11.017.

28. Gizem Özbaykal, Eva Wollrab, Francois Simon, Antoine Vigouroux, Baptiste Cordier, Andrey Aristov, Thibault Chaze, Mariette Matondo, and Sven van Teeffelen. The transpeptidase PBP2 governs initial localization and activity of the major cell-wall synthesis machinery in E. coli. eLife, 9:1-37, 2020. ISSN 2050084X. doi: 10.7554/eLife.50629.

29. Bing Liu, Logan Persons, Lynda Lee, and Piet A.J. de Boer. Roles for both FtsA and the FtsBLQ subcomplex in FtsN-stimulated cell constriction in Escherichia coli. Molecular Microbiology, 95(6):945-970, 2015. ISSN 13652958. doi: 10.1111/mmi.12906.

30. Mary Jane Tsang and Thomas G. Bernhardt. A role for the FtsQLB complex in cytokinetic ring activation revealed by an $\mathrm{fts} L$ allele that accelerates division. Molecular Microbiology, 95(6):925-944, 2015. ISSN 13652958. doi: 10.1111/mmi.12905.

31. Michael Tarry, S. J.Ryan Arends, Pietro Roversi, Evan Piette, Frank Sargent, Ben C. Berks, 
bioRxiv preprint doi: https://doi.org/10.1101/850073; this version posted September 29, 2020. The copyright holder for this preprint (which was not certified by peer review) is the author/funder, who has granted bioRxiv a license to display the preprint in perpetuity. It is made available under aCC-BY-NC-ND 4.0 International license.

David S. Weiss, and Susan M. Lea. The Escherichia coli Cell Division Protein and Model Tat Substrate Sufl (FtsP) Localizes to the Septal Ring and Has a Multicopper Oxidase-Like Structure. Journal of Molecular Biology, 386(2):504-519, 2009. ISSN 00222836. doi: 10.1016/j.jmb.2008.12.043.

32. Waldemar Vollmer and Stephen J. Seligman. Architecture of peptidoglycan: more data and more models. Trends in Microbiology, 18(2):59-66, 2010. ISSN 0966842X. doi: 10.1016/j. tim.2009.12.004.

33. L. G. Burman and J. T. Park. Molecular model for elongation of the murein sacculus of Escherichia coli. Proceedings of the National Academy of Sciences of the United States of America, 81(6 I):1844-1848, 1984. ISSN 00278424. doi: 10.1073/pnas.81.6.1844. 